\title{
Spatial fluctuations of optical solitons due to long-range correlated dielectric perturbations in liquid crystals
}

\author{
Serena Bolis, ${ }^{1,2, *}$ Simon-Pierre Gorza, ${ }^{1}$ Steve J. Elston, ${ }^{3}$ Kristiaan Neyts, ${ }^{2}$ Pascal Kockaert, ${ }^{1}$ and Jeroen Beeckman ${ }^{2}$ \\ ${ }^{1}$ Université libre de Bruxelles, OPERA-Photonics Group, 50 Avenue F.D. Roosevelt, Code Postal 194/5, 1050 Bruxelles, Belgium \\ ${ }^{2}$ ELIS Department, Gent Universiteit, Technologiepark-Zwijnaarde 15, 9052 Gent, Belgium \\ ${ }^{3}$ Department of Engineering Science, University of Oxford, Parks Road, Oxford OX1 3PJ, United Kingdom
}

(Received 13 August 2017; published 29 September 2017)

\begin{abstract}
Starting from the de Gennes theory of director fluctuations in nematics, we report on a model of the spatial fluctuations in nematicon propagation. We demonstrate that, when the long-range correlation that characterizes nematic liquid crystals is taken into account in the thermal noise, it is possible to account for the spatial oscillations and propagation losses experienced by nematicons. Increasing the power of the nematicon, the oscillation amplitudes increase and the propagation losses decrease. The nematicon is then more strongly confined and deviates more, but is less scattered by the thermally induced perturbations of the refractive index. All the results are in good agreement with the experimental observations.
\end{abstract}

DOI: 10.1103/PhysRevA.96.031803

\section{INTRODUCTION}

Liquid crystals (LCs) are a particular phase of matter which is between the solid and the liquid states. Their long-range order, combined with their molecular optical anisotropy, gives rise to a macroscopic birefringence. Moreover, their viscosity allows the molecules to flow one over the other which makes them very sensitive to external stimuli (mechanical, electrical,...) [1]. For these reasons, when an optical beam is injected into a cell filled with nematic LCs with positive anisotropy, the LC molecules are reoriented along the optical electric field, inducing a local increase of the refractive index. The so-created optical waveguide can prevent the diffraction of the beam, which propagates maintaining its transverse intensity profile. This solitonlike propagation is termed a nematicon, as it occurs in nematic liquid crystals [2-7].

Nematic LCs are characterized by a macroscopic mean orientation of the LC molecules, called the director and represented by the unitary vector $\vec{n}_{0}$. Thermal noise induces local variations of the director, described by the functions $n_{\hat{x}}(\vec{r}), n_{\hat{y}}(\vec{r})$ and whose average is zero. These fluctuations, which cannot be too abrupt in space due to elastic restoring forces between the molecules, are well described by the de Gennes theory $[1,8]$. Starting from the Oseen-Frank continuum theory for LCs, de Gennes theory predicts the value of the spatial correlation of the director fluctuations. These long-range fluctuations are responsible for optical scattering in liquid-crystal materials. This scattering is six orders of magnitude larger than in conventional isotropic fluids which are dominated by the Rayleigh scattering [1] and is the main contribution to optical propagation losses in liquid crystals [9].

From the de Gennes theory, it is possible to extract the variance of the molecular fluctuations, which can be used to predict nuclear spin fluctuations [10], asymmetry in electron resonance spectra [11], and to define the order parameter that characterizes nematic LCs [1]. The off-diagonal elements of the covariance matrix described by de Gennes take into account the long-range interactions of the molecules and the

\footnotetext{
*serena.bolis@ulb.ac.be
}

spatial correlation of the director within the LC cell. These correlations are often neglected when considering director fluctuations in liquid crystals.

The proper modeling of the director fluctuations is of particular importance for the understanding of phenomena that depend on noise, such as modulation instability $[12,13]$, filamentation [14], or speckle formation [15,16]. Also, the thermally induced refractive index fluctuations are responsible for soliton spatial fluctuations. Indeed, when the power is high enough to induce the nematicon diameter to be of the same order of magnitude as or smaller than the refractive index grains, nematicon spatial fluctuations are observed $[17,18]$. Different ways to quench the fluctuations have been proposed, ranging from applying an external electric field [19] to the polymerization of the medium [20].

In this work we explicitly make use of the full correlation matrix in our simulations to generate a model of long-range correlated noise starting from the elastic constants of the LCs and the thermal energy of the system. First, we consider the linear propagation regime in order to explain the origin of speckle formation. Then the nonlinear propagation and the soliton formation are analyzed and our model is experimentally verified. Our results demonstrate that consideration of the correlation is crucial to explain the origin of the nematicon fluctuations.

\section{ANALYTIC AND NUMERICAL MODELING}

\section{A. Geometry of the system}

The geometry of the LC cell is similar to previous works [21]. It consists of two substrates with planar alignment and a rubbing direction of $\theta_{0}=45^{\circ}$ with respect to the $z$ axis and a cell gap of $75 \mu \mathrm{m}$ [Fig. 1(a)]. It is filled with a solution of $1 \mathrm{wt} \%$ of pyrromethene 597 dye (PM597, Sigma-Aldrich) in E7 nematic LC (Merck). Note that for this study the presence of the dye is not relevant since it does not absorb the cw $1064 \mathrm{~nm}$ infrared light of the laser used to excite the nematicons. The light is injected into the cell through an optical fiber slid between the two substrates, with a cladding diameter of $64.4 \mu \mathrm{m}$, a core diameter of $2.9 \mu \mathrm{m}$, and a cut-off wavelength of $550 \mathrm{~nm}$. 
(a)

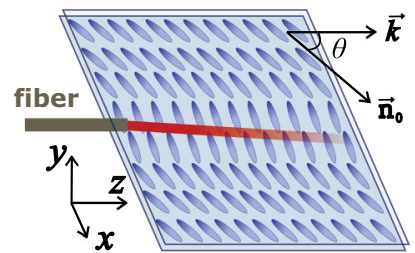

(b)

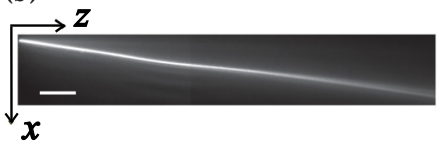

(c)

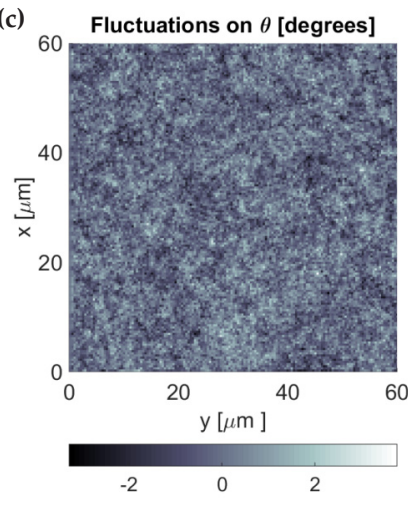

FIG. 1. (a) Schematic of the nematicon injection into the LC cell through an optical fiber. (b) Nematicon profile generated for a beam of $3.7 \mathrm{~mW}$ at $23 \mathrm{C}^{\circ}$; the scale bar is $500 \mu \mathrm{m}$. (c) Example of generated correlated noise.

The injected mode is thus almost Gaussian with a radius of $3.7 \mu \mathrm{m}$. The phase front is assumed to be planar at the exit of the fiber.

\section{B. Analytic and numerical model}

We consider a monochromatic optical beam with a slowly varying envelope $\mathcal{A}(\vec{r})$ propagating in the medium with the wave vector $\vec{k}$ parallel to the $z$ axis in the paraxial approximation. We also assume the light to be polarized along the $x$ axis (scalar approximation). With these assumptions the propagation equation reads $[4,17]$

$$
\begin{aligned}
2 i k_{0} n_{r} \frac{\partial \mathcal{A}}{\partial z}= & \Delta_{\perp} \mathcal{A}+\left[k_{0}^{2} \Delta \varepsilon\left(\sin ^{2} \theta-\sin ^{2} \theta_{0}\right)\right] \mathcal{A} \\
& +2 i k_{0} n_{r} \tan \delta(\theta) \frac{\partial \mathcal{A}}{\partial x}
\end{aligned}
$$

where $\theta=\theta(\vec{r})$ is the angle between $\vec{k}$ and the director $\vec{n}_{0}, \Delta \varepsilon=\varepsilon_{\|}-\varepsilon_{\perp}$ is the dielectric anisotropy at optical frequencies, $n_{r}=\sqrt{\varepsilon_{\perp}+\Delta \varepsilon \sin ^{2}\left(\theta_{0}\right)}$ is the refractive index for input light (where the molecules are at $\theta=\theta_{0}$ ), $\Delta_{\perp}$ is the transverse Laplacian operator, and $k_{0}=2 \pi / \lambda$ is the wave number in vacuum. The last term of Eq. (1) takes into account the effect of the walk-off angle $\delta(\theta)$ arising from the anisotropy of the medium and defined as $[2,5]$

$$
\tan \delta(\theta)=\frac{\Delta \varepsilon \sin \theta \cos \theta}{\varepsilon_{\perp}+\Delta \varepsilon \cos ^{2} \theta} .
$$

The nonlinear response of the LC can be described by the Euler-Lagrange theory in the one-constant approximation [2]

$$
K \Delta_{\perp} \theta+\frac{1}{2} \varepsilon_{0} \sin \{2[\theta-\delta(\theta)]\} \Delta \varepsilon|\mathcal{A}|^{2}=0,
$$

where $K$ is the elastic constant of the LC.

The system of coupled equations (1) and (3) is solved as follows. Discretizing in the $z$ direction, at step $N$, the equilibrium distribution $\theta\left(x, y, z_{N}\right)$ of the molecules is found by integrating Eq. (3) for the field $\mathcal{A}\left(x, y, z_{N}\right)$. Equation (1) is then solved with this molecular distribution to compute the field distribution at step $N+1$. Equation (1) is integrated using a beam propagation method (BPM) along $z$, while Eq. (3) is

solved with the Newton-Raphson method [22]. Both Eqs. (1) and (3) are discretized with a Crank-Nicolson method, in order to conserve the energy of the propagating beam [22].

Finally, note that a coordinate transformation is used which effectively tilts the frame of reference by an angle $\delta\left(\theta_{0}\right)$ with respect to $z$ in order to decrease the numerical errors [23]. This change of coordinates $x^{\prime}=x+z \tan \delta\left(\theta_{0}\right), y^{\prime}=y$, and $z^{\prime}=z$ decreases the amplitude of the walk-off term, without affecting the term $\Delta_{\perp} \mathcal{A}$ or changing the orientation of the electric field polarization.

\section{Correlated noise}

Experimentally, it has been observed that high power nematicons show fluctuations in their spatial trajectory [Fig. 1(b)]. This has been explained as being due to a deflection of the soliton direction with refractive index perturbations when the soliton diameter becomes comparable to the typical size of the refractive index perturbations $[17,18,20]$.

In the modeling presented above, the noise is not yet taken into account. As the refractive index perturbations originate from the thermal noise, the model of Eqs. (1) and (3) cannot be used directly to account for the soliton fluctuations.

The fluctuation theory for nematics was originally developed by de Gennes $[1,8,24]$. In his work, starting from the Oseen-Frank continuum theory for LCs, he expressed the free-energy contribution due to the director fluctuations $\left(n_{\hat{x}}, n_{\hat{y}}\right)$ in the frame of reference $(\hat{x}, \hat{y}, \hat{z})$, where the axis $\hat{z}$ lies along the director $\vec{n}_{0}$. Then he evaluated the average of these fluctuations using the equipartition theorem from statistical mechanics. The expression for the correlation of $n_{\alpha}(\alpha=\hat{x}, \hat{y})$ between two points $\overrightarrow{r_{1}}$ and $\overrightarrow{r_{2}}$ separated by $\vec{R}$, when a low-frequency external electric field $E_{\text {ext }}$ is applied along $\hat{z}$, is given by the expression

$$
\left\langle n_{\alpha}\left(\vec{r}_{1}\right) n_{\alpha}\left(\vec{r}_{1}+\vec{R}\right)\right\rangle=\frac{k_{B} T}{K(2 \pi)^{3}} \int_{0}^{q_{\max }} \frac{\exp (i \vec{q} \cdot \vec{R})}{q^{2}+\xi^{-2}} d \vec{q},
$$

where $\langle\cdot\rangle$ denotes the thermal average from the equipartition theorem, the components of $\vec{q}$ are $q_{\beta}=2 \pi / \beta$ with $\beta=\hat{x}, \hat{y}, \hat{z}$, and $\xi=K /\left(\varepsilon_{0} \Delta \varepsilon\left|E_{\text {ext }}\right|^{2}\right)$ is the so-called coherence length. The integral is limited between zero and $q_{\max }=2 \pi / a$, where $a$ is the smallest size below which the continuum theory is no longer valid. de Gennes showed that, in the limit $\|\vec{R}\| \gg a$, Eq. (4) simplifies to

$$
\left\langle n_{\alpha}\left(\vec{r}_{1}\right) n_{\alpha}\left(\vec{r}_{1}+\vec{R}\right)\right\rangle=\frac{k_{B} T}{4 \pi K R} \exp (-R / \xi) .
$$

This equation shows the truly long-range $(\propto 1 / R)$ correlation in nematic LCs, which is exponentially damped in the presence of an electric field.

Equation (4) describes the covariance matrix, where the diagonal elements, obtained for $\vec{R} \rightarrow 0$, correspond to the variance of the fluctuations around the director

$$
\left\langle n_{\alpha}^{2}\right\rangle=\frac{k_{B} T}{2 \pi^{2} K}\left(q_{\max }-\frac{\pi}{2 \xi}\right),
$$

which can be used to evaluate the order parameter of the system $[10,25]$. As $q_{\max }=2 \pi / a$, the proper choice of $a$ is crucial in order to evaluate the amplitude of the fluctuations 
and it is usually taken to be of the order of magnitude of the intermolecular $[1,11]$ or intermicellar [10] distance, values below which the continuum theory of LCs is no longer valid.

The values of $\vec{R}$ in Eq. (4) are limited to the points on the discretization grid. Since this grid $(\Delta x=\Delta y=355 \mathrm{~nm}$, $\Delta z=900 \mathrm{~nm}$ ) is much larger than the intermolecular distance (a few nanometers) due to computing memory limitations, the correlation is greatly decreased over the grid length scales $\Delta x$, $\Delta y$, and $\Delta z$, and the diagonal elements of the matrix calculated with Eq. (6) would be much larger than the off-axis elements described by Eq. (5). Such an almost-diagonal matrix would then describe a largely uncorrelated system, and we would lose the benefit of our approach, as will be shown later. For this reason we take as diagonal elements the average $A$ of the correlation function over one grid unit size, i.e., along $x$ :

$$
\begin{aligned}
A & {\left[\left\langle n_{\alpha}\left(\vec{r}_{1}\right) n_{\alpha}\left(\vec{r}_{1}+\vec{R}\right)\right\rangle\right] } \\
& =\frac{k_{B} T}{K(2 \pi)^{3}} \frac{1}{\Delta x} \int_{0}^{\Delta x} \int_{0}^{q_{\max }} \frac{\exp (i \vec{q} \cdot \vec{R})}{\left(q^{2}+\xi^{-2}\right)} d \vec{q} d R .
\end{aligned}
$$

Taking, in our case, the grid size as the limit of the continuum theory $\left(q_{\max }=2 \pi / \Delta x\right)$ and introducing the definition of the sine integral $\operatorname{Si}(x)=\int_{0}^{x} \frac{\sin t}{t} d t$, we obtain

$$
\begin{aligned}
A & {\left[\left\langle n_{\alpha}\left(\vec{r}_{1}\right) n_{\alpha}\left(\vec{r}_{1}+\vec{R}\right)\right\rangle\right] } \\
& =\frac{k_{B} T}{2 K \pi^{2}} \frac{1}{\Delta x} \int_{0}^{2 \pi / \Delta x} \frac{q}{\left(q^{2}+\xi^{-2}\right)} \operatorname{Si}(q \Delta x) d q,
\end{aligned}
$$

which constitutes the value on the diagonal elements of the covariance matrix.

Using Eq. (8) and Eq. (5), respectively, for the diagonal and off-diagonal elements, it is possible to generate the correlation matrix $\overline{\bar{C}}$. An uncorrelated random variable $\overline{\bar{N}}_{\text {uncorr }}$ normally distributed is generated and the correlation is forced through the relation $\overline{\bar{N}}_{\text {corr }}=\overline{\bar{U}} \overline{\bar{N}}_{\text {uncorr }}$, where $\overline{\bar{N}}_{\text {corr }}$ is the correlated noise and $\overline{\bar{U}}$ is the upper triangular form of the Cholesky decomposition of the correlation matrix [26].

Due to the large volume covered by the soliton propagation and the finite availability of computational memory, it is not possible to write the complete three-dimensional correlation matrix, as it scales with the square of the number of points. Given the geometry of the system (BPM and one-constant approximation), we generate the correlated noise in planes $x y$ orthogonal to the propagation.

Since the variable that describes the LC molecules is $\theta$, we choose one of the two fluctuation components, $n_{\hat{x}}$, to be in the plane $x z$. In this way, the angular variation $d \theta$ can easily be calculated with $d \theta=\arctan \left(n_{\hat{x}}\right)$ and added to $\theta$ after the integration of Eq. (3). A typical noise pattern obtained by this method is shown in Fig. 1(c), where the slowly varying oscillations of the angle $\theta$ can clearly be seen.

The $x y$-correlated noise is not generated at every step, since it would mean a completely uncorrelated noise along $z$, but every $10 \mu \mathrm{m}$, which is the same order of magnitude as the grains in the correlated noise pattern. The noise is then linearly interpolated along $z$, in order to have a noise profile slowly varying along $z$, with an appropriate level of correlation in this direction. Finally, since we do not apply a homogeneous electric field to the cell and since the peak of the optical electric
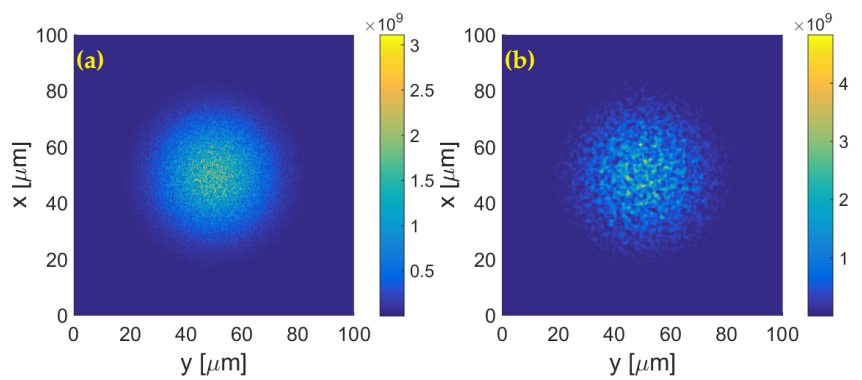

FIG. 2. Transmission of visible (532 nm) light through a 50- $\mu \mathrm{m}$ thick cell for the case of uncorrelated (a) and correlated (b) thermal noise.

field is too small to induce a significant quenching of the director fluctuations [27], in the simulations we consider $E_{\text {ext }}$ null and hence $\xi$ infinite.

\section{RESULTS AND DISCUSSION}

\section{A. Linear propagation}

We will start analyzing the light propagation for powers low enough to avoid any nonlinear effects. We thus expect to reproduce by numerical simulations the observed so-called speckle pattern produced when a laser beam propagates through a liquid-crystal device. In order to compare the results with the literature $[15,16]$, we simulate the propagation of visible light $(532 \mathrm{~nm})$ through a planar aligned $50-\mu \mathrm{m}$ cell filled with E7. The parameters used for E7 are $K=12 \mathrm{pN}$ and $\varepsilon_{\|}=2.9204, \varepsilon_{\perp}=2.2681$ [28]. The effect of the spatial correlations will be highlighted by comparing the propagation with noise which is uncorrelated [Fig. 2(a)] and noise which is correlated [Fig. 2(b)].

For the case of noise which is uncorrelated, the noise is randomly generated at every step along the propagation. The resulting output is an almost unperturbed beam profile [Fig. 2(a)]. As the noise grain size equals the simulation grid, which is smaller than the wavelength, the light cannot be locally focused. It therefore follows a homogeneous output profile without any speckle pattern. Also, the noise-induced diffraction is averaged along $z$; since the director fluctuation is generated at every simulation step, this effectively removes any correlated-like pattern along the propagation.

For the case of noise which is correlated, the smooth variation of the director distribution (and therefore of the refractive index) causes a local (random) focusing and defocusing of the light as it propagates and the characteristic speckle pattern is therefore generated [Fig. 2(b)]. We have thus shown that the spatial correlation of the LC director is essential to account for the characteristic speckle generation in LCs.

\section{B. Experimental nematicon propagation}

Experimental observations of the spatial fluctuations of a soliton propagating in the sample described in Sec. II A are undertaken. The IR soliton is injected in the plane of the cell as described in Sec. II A and it propagates a few millimeters. The acquired intensity profiles are similar to the one shown in Fig. 1(b) for the case of $3.7 \mathrm{~mW}$, with noise-induced 

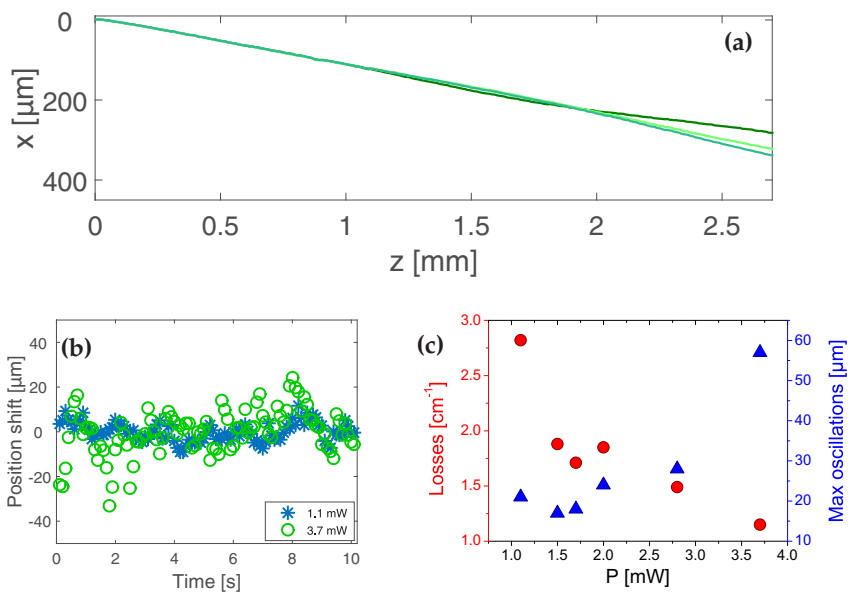

FIG. 3. (a) Experimental soliton trajectory for an input power of $3.7 \mathrm{~mW}$. (b) Position of the beam after $2.7 \mathrm{~mm}$ of propagation as a function of time for a soliton of $1.1 \mathrm{~mW}$ (stars) and of $3.7 \mathrm{~mW}$ (empty circles). (c) Oscillation amplitudes (blue triangles, right axis) and losses (red circles, left axis) as a function of the power.

fluctuations. We estimate the soliton trajectory along the propagation distance by interpolating, at different position $z$, the measured intensity profile along $x$ by a Gaussian function. The soliton propagation paths for three different acquisitions are shown in Fig. 3(a).

Since the LC molecules fluctuate due to thermal noise, the refractive index profile and therefore the soliton path vary in time. Plotting the soliton position as a function of time after $2.7 \mathrm{~mm}$ of propagation for two different powers [Fig. 3(b)] shows that the soliton oscillates around a mean value and that the largest oscillations appear at the highest power. In Fig. 3(c) (blue triangles) we plot the amplitude of the oscillations as a function of the power. The oscillations increase by almost a factor of 3 between 1.1 and $3.7 \mathrm{~mW}$. This can be explained by the fact that increasing the power, the nematicon undergoes a transition to a breathing propagation regime, where the beam becomes overfocused periodically $[29,30]$. Indeed, when the soliton diameter becomes smaller than the director fluctuation grain size, the nematicon, instead of being diffracted and destroyed, is rather refracted [31] and hence its propagation path deviates.

From the Gaussian fit to the measured intensity profile along $x$ it is also possible to compute the integral of the intensity profile, in order to obtain the evolution of the energy as a function of $z$. The exponential decay is fitted with a function $\propto \exp (-\alpha z)$, where $\alpha$ represents the loss coefficient. The evolution of $\alpha$ as a function of the power is also shown in Fig. 3(c) (red circles). Increasing the power, and therefore the focusing of the soliton, we also observe a decrease of the loss coefficient as the photons are less scattered. This is consistent with the fact that the soliton is refracted (leading to propagation path deviation), rather than scattered, by the refractive index perturbations.

\section{Numerical nematicon propagation}

We performed 50 simulations of the propagation of solitons for input powers ranging from 1.1 to $3.7 \mathrm{~mW}$. For every
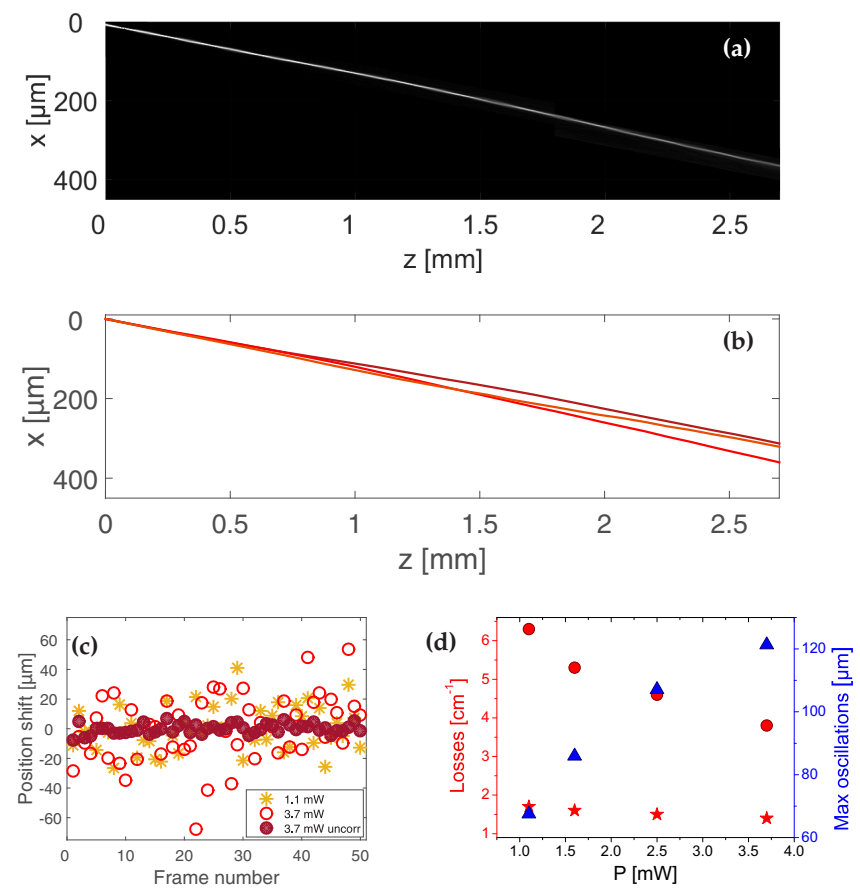

FIG. 4. (a) Density plot of the intensity for one of the simulations of a 3.7-mW soliton. (b) Numerical simulation of the soliton trajectory for three different simulations with a soliton power of $3.7 \mathrm{~mW}$. (c) Position of the beam after $2.7 \mathrm{~mm}$ of propagation for 50 generations of the noise for a soliton of $1.1 \mathrm{~mW}$ (stars), $3.7 \mathrm{~mW}$ (empty circles), and $3.7 \mathrm{~mW}$ for the case of uncorrelated noise (full circles). (d) Oscillation amplitudes (blue triangles, right axis) and losses (left axis) as a function of the power. The losses are plotted for the case of correlated (circles) and uncorrelated (stars) noise.

simulation a different correlated director noise pattern is generated every $10 \mu \mathrm{m}$, as described in Sec. II C. In Fig. 4(a) we show one soliton intensity profile for $3.7 \mathrm{~mW}$, while examples of soliton trajectories at the same power are shown in Fig. 4(b). Even if abrupt changes in the path direction are not present, the spatial oscillations obtained in the numerical simulations are in good agreement with the experimental results.

The nematicon position after $2.7 \mathrm{~mm}$ of propagation is shown in Fig. 4(c) for 1.1 and $3.7 \mathrm{~mW}$. Since our model does not describe the temporal behavior of the system, every simulation is independent of the previous one. For this reason, when we represent the oscillation amplitudes at different times (referred to as frames here), we do not observe a continuous evolution as in the experiments. However, comparing the maximum deviation of the soliton from the straight path, we observe larger oscillations for increasing powers, consistent with the experimental observations.

Importantly, comparing models of the soliton oscillations at high power for cases where the modeled noise is correlated and uncorrelated [Fig. 4(c)], we observe a drop in the nematicon oscillation amplitudes from $\sim 120 \mu \mathrm{m}$ to $\sim 15 \mu \mathrm{m}$ in the latter case. Without the spatial correlation of the director fluctuations, the length scale of the refractive index variations is much smaller than the soliton diameter. In practice the length scale of these variations is then defined by the discretization grid used for the simulations. In that limit, the soliton only 
slightly deviates during propagation even at high powers. The spatial correlation of the director fluctuations is therefore an important consideration for the proper modeling of the spatial fluctuations of soliton propagation in LCs.

The correlated noise in the numerical modeling causes a power-dependent behavior of the oscillation amplitudes [Fig. 4(d), blue triangles] that is in good agreement with the experimentally observed behavior. The difference between the experimental results and the simulations with correlated noise can be explained by the fact that in practice, when the fiber is slid into the LC cell, it causes a perturbation in the LC director profile that is not taken into account in our model. This perturbation extends over a distance that is multiple times the thickness of the cell and therefore causes a delay in the formation of the soliton equilibrium profile [32]. For this reason, in the experiment, the nematicon does not oscillate from the beginning of its propagation [Fig. 3(a)]. We therefore measure the oscillation amplitudes at a distance that is effectively shorter than $2.7 \mathrm{~mm}$. The amplitudes experimentally measured are therefore smaller than the ones obtained from the simulations, where the soliton starts to oscillate close to the injection. Also, in our one-constant approximation, we assume the correlation to be isotropic. However, along the $z$ axis, a dominant bend-splay deformation takes place, which may be associated with a larger elastic constant $\left(K_{3}=19.5 \mathrm{pN}\right.$, [5]). Since the correlation is inversely proportional to the elastic constant $K$, we are overestimating the correlation and therefore the scattering as the nematicon propagates.

In Fig. 4(d) (red circles), we show the loss coefficient $\alpha$ as a function of the input power for the case of correlated noise. The values and the power-dependent behavior of $\alpha$, ranging from 6.3 to $3.8 \mathrm{~cm}^{-1}$ over our range of powers, are in good agreement with the experiments. The factor 2 of difference may be due to the overestimation of the scattering in the oneconstant approximation. The same order of magnitude can be found in the literature $[2,33]$, even if no dependence on the nematicon power is reported there. On the same graph, as a comparison, we also show the losses obtained for the case of uncorrelated noise, for which almost no dependence on the nematicon power is observed. The losses are substantially less $\left(\sim 1.5 \mathrm{~cm}^{-1}\right)$ when the director fluctuation noise is uncorrelated since the soliton does not experience the effects of speckle formation (as observed in Fig. 2) and there is consequently no loss of energy in the central part of the beam. The losses are also almost independent of the nematicon power, showing once again how taking into account the correlation in director fluctuations improves the description of soliton propagation in liquid-crystalline systems.

\section{CONCLUSIONS}

We have demonstrated a method to properly include the thermal fluctuations of the director in LCs when modeling nematicon propagation, starting from the common parameters of LCs. In such systems, the long-range interaction among the molecules must be taken into account when generating the thermal noise, through the spatial correlation of the director fluctuations. The computed correlated noise allows us to explain the experimentally observed spatial fluctuations of nematicons at high powers. Further improvements could be added to our model, for example through the introduction of full modeling of the correlation along the $z$ direction or the temporal evolution of the director orientation, the power dependence of the loss coefficient, and the introduction of the three elastic constants. However, the oscillation amplitudes obtained with our model are in good agreement with the values experimentally measured. We believe that this method will also help the modeling of both linear phenomena involving speckle generation and more complex nonlinear behavior in LCs, such as modulation instabilities or filamentation.

\section{ACKNOWLEDGMENTS}

We would like to acknowledge support from the Belgian Science Policy Office (BELSPO) under Grant No. IAP7-35, the Fondation Philippe Wiener-Maurice Anspach, the Fund for Research Training in Industry and Agriculture (FRIA), the Fonds David et Alice Van Buuren, and the Fondation JaumotteDemoulin. S.B. thanks T. Bury for the valuable discussions.
[1] P. G. de Gennes and J. Prost, The Physics of Liquid Crystals, 2nd ed. (Oxford University Press, New York, 1995).

[2] A. Alberucci, A. Piccardi, M. Peccianti, M. Kaczmarek, and G. Assanto, Phys. Rev. A 82, 023806 (2010).

[3] A. Alberucci and G. Assanto, Phys. Rev. A 83, 033822 (2011).

[4] M. Peccianti, A. D. Rossi, G. Assanto, A. D. Luca, C. Umeton, and I. Khoo, Appl. Phys. Lett. 77, 7 (2000).

[5] M. Peccianti, A. Fratalocchi, and G. Assanto, Opt. Express 12, 6524 (2004).

[6] M. Peccianti, C. Conti, G. Assanto, A. De Luca, and C. Umeton, Nature (London) 432, 733 (2004).

[7] J. Beeckman, H. Azarinia, and M. Haelterman, Opt. Lett. 34, 1900 (2009).

[8] P. G. de Gennes, C. R. Acad. Sci. Paris 266, 15 (1968).

[9] S.-T. Wu and K.-C. Lim, Appl. Opt. 26, 1722 (1987).

[10] B. Halle, P. O. Quist, and I. Furó, Phys. Rev. A 45, 3763 (1992).
[11] S. Brooks, G. Luckhurst, and G. Pedulli, Chem. Phys. Lett. 11, 159 (1971).

[12] M. Peccianti, C. Conti, and G. Assanto, Phys. Rev. E 68, 025602 (2003).

[13] J. Beeckman, X. Hutsebaut, M. Haelterman, and K. Neyts, Opt. Express 15, 11185 (2007).

[14] M. Peccianti, C. Conti, and G. Assanto, Opt. Lett. 28, 2231 (2003).

[15] G. Agez, P. Glorieux, C. Szwaj, and E. Louvergneaux, Opt. Commun. 245, 243 (2005).

[16] H. Orihara, N. Sakurai, Y. Sasaki, and T. Nagaya, Phys. Rev. E 95, 042705 (2017).

[17] J. Beeckman, K. Neyts, X. Hutsebaut, C. Cambournac, and M. Haelterman, Opt. Express 12, 1011 (2004).

[18] A. I. Strinić, D. V. Timotijević, D. Arsenović, M. S. Petrović, and M. R. Belić, Opt. Express 13, 493 (2005). 
[19] J. Henninot, J. Blach, and M. Warenghem, J. Opt. A: Pure Appl. Opt. 10, 085104 (2008).

[20] N. Karimi, A. Alberucci, M. Virkki, A. Priimagi, M. Kauranen, and G. Assanto, Photonics Lett. Pol. 8, 2 (2016).

[21] S. Bolis, T. Virgili, S. K. Rajendran, J. Beeckman, and P. Kockaert, Opt. Lett. 41, 2245 (2016).

[22] W. Press, B. Flannery, S. Teukolsky, and W. Vetterling, Numerical Recipes in C: The Art of Scientific Computing, 2nd ed. (Cambridge University Press, Cambridge, UK, 1992).

[23] G. Agrawal, Nonlinear Fiber Optics, 4th ed. (Academic, New York, 2007).

[24] P. G. de Gennes, Mol. Cryst. Liq. Cryst. 7, 325 (1969).

[25] B. Halle, Liq. Cryst. 17, 759 (1994).

[26] A. Briggs, M. Sculpher, and K. Claxton, Decision Modeling for Health Economic Evaluation (Oxford University Press, Oxford, 2006).
[27] I. Lelidis, M. Nobili, and G. Durand, Phys. Rev. E 48, 3818 (1993).

[28] J. Li, S.-T. Wu, S. Brugioni, R. Meucci, and S. Faetti, J. Appl. Phys. 97, 073501 (2005).

[29] C. Conti, M. Peccianti, and G. Assanto, Phys. Rev. Lett. 92, 113902 (2004).

[30] X. Hutsebaut, C. Cambournac, M. Haelterman, J. Beeckman, and K. Neyts, J. Opt. Soc. Am. B 22, 1424 (2005).

[31] A. Alberucci, G. Assanto, A. A. Minzoni, and N. F. Smyth, Phys. Rev. A 85, 013804 (2012).

[32] J. Beeckman, Lateral light propagation and spatial optical solitons in liquid crystal devices, Ph.D. thesis, Universiteit Gent, 2005-2006.

[33] N. Karimi, A. Alberucci, O. Buchnev, M. Virkki, M. Kauranen, and G. Assanto, J Opt. Soc. Am. B 33, 903 (2016). 